Cahiers $d u$ MONDE RUSSE

\section{Cahiers du monde russe}

Russie - Empire russe - Union soviétique et États indépendants

$58 / 4 \mid 2017$

Varia

\title{
Matthew P. Romaniello, Tricia Starks, eds., Russian History through the Senses: From 1700 to Present
}

\section{Magali Delaloye}

\section{(2penEdition}

Journals

Édition électronique

URL : http://journals.openedition.org/monderusse/10210

DOI : $10.4000 /$ monderusse. 10210

ISSN : $1777-5388$

Éditeur

Éditions de l'EHESS

\section{Édition imprimée}

Date de publication : 1 octobre 2017

Pagination : 768-770

ISBN : 978-2-7132-2698-4

ISSN : $1252-6576$

\section{Référence électronique}

Magali Delaloye, « Matthew P. Romaniello, Tricia Starks, eds., Russian History through the Senses: From 1700 to Present », Cahiers du monde russe [En ligne], 58/4 | 2017, mis en ligne le 01 octobre 2017, consulté le 08 janvier 2021. URL : http://journals.openedition.org/monderusse/10210 ; DOI : https://doi.org/10.4000/monderusse. 10210 
Matthew P. ROMANIELLO, Tricia STARKS, eds.,

\section{Russian History through the Senses \\ From 1700 to Present}

Londres - New York : Bloomsbury, 2016, 320 p.

Ces dernières années, l'historiographie connaît ce qu'on pourrait appeler un « tournant sensitif ». Nombre d'historiens et historiennes suivent désormais la voie ouverte par le pionnier de cette approche, Alain Corbin, qui, en 1982 déjà, montrait l'apport de la prise en compte des sens - en particulier l'odeur - dans l'histoire culturelle, socio-économique mais également de la médecine et de l'urbanisme ${ }^{1}$. Cette approche a connu un regain d'intérêt dès le milieu des années 2000, donnant naissance notamment à la somme en six volumes $A$ Cultural History of the Senses ${ }^{2}$. C'est dans cette lignée que se situe ce recueil de contributions édité par Matthew Romaniello et Tricia Starks, tous deux professeurs, l'un à l'université d'Hawaï, l'autre à l'université de l'Arkansas, et spécialistes de l'histoire culturelle russe ${ }^{3}$. $\mathrm{Nul}$ doute que ce livre apporte une contribution majeure à un renouvellement de la recherche sur l'histoire culturelle russe ${ }^{4}$. Après le corps et les émotions, les sens représentent une composante essentielle de l'analyse de la construction des subjectivités, mais aussi des identités collectives : c'est par eux que les individus font l'expérience du monde social. À travers les thèmes les plus variés, en ne se réduisant pas à un seul sens, ce livre offre, en outre, des pistes théoriques qui permettront la continuation de cette voie historiographique.

L'ouvrage s'ouvre sur une introduction très complète d'Alexander Martin (chapitre 1) qui propose une réflexion sur la question des sens dans les cultures russe et soviétique. En premier lieu, il situe les réflexions de ce livre dans les divers contextes historiographiques dans lesquels s'inscrivent les contributions. Il en met cinq en exergue : l'analyse du capitalisme et du consumérisme par Walter Benjamin ; la réflexion de Norbert Elias à propos du processus de civilisation et du lien entre les sens et le pouvoir; la réflexion de Michel Foucault sur les formes de pouvoir institutionnel sur le corps des citoyens ; les recherches sur la construction des identités collectives ; et, pour finir, celles traitant de la question de la mémoire, individuelle ou collective. Ces champs historiographiques sont renouvelés par la réflexion sur le rôle des sens. Enfin, alors que le livre est séparé en quatre parties chronologiques - Russie impériale, Russie révolutionnaire, Russie soviétique et la question de la mémoire dans la Russie postsoviétique -, l'auteur propose un autre découpage possible - qui sera repris dans ce compte rendu -, selon les apports historiographiques des contributions. Un premier groupe d'articles permettent de questionner le rôle des sens dans les perceptions de soi et la construction des identités collectives. Une deuxième série de contributions analysent les politiques d'État pour changer les comportements des individus et, ainsi, montrent le rôle des sens dans les ingéniéries sociales mises en place. Enfin, un dernier groupe de textes portent sur une réflexion concernant l'expérience sensorielle des guerres (p. 13).

Commençons par les contributions qui saisissent les sens pour comprendre la construction des identités collectives. Dans cette catégorie, pour la période impériale, 
Alison K. Smith (chapitre 3) se penche sur la question de la différence entre fermentation et putréfaction - donc entre le «bon» et le «mauvais » - et, en prenant l'exemple du kvas, le rôle du goût dans cette construction qui donne une cohérence et un ordre sociaux. Pour la période révolutionnaire, Tricia Starks (chapitre 5), analyse, elle, l'utilisation du tabac, cherchant à retrouver le goût et l'odeur de la cigarette qui devient un véritable marqueur social. Toujours pour la période révolutionnaire, Aaron B. Retish (chapitre 7) développe le débat autour de la kumyška, boisson alcoolisée traditionnelle chez le peuple finno-ougrien des Oudmourtes. Construite culturellement par les élites russes comme «primitive», cette boisson devient un « ennemi du peuple » durant la Guerre civile qui prône l'accès à la modernité.

Les sens ont également un rôle dans l'ingéniérie sociale mise en place par les différents gouvernements tout au long de l'histoire russe, comme le montrent les contributions de ce deuxième groupe de textes. Abby Schrader (chapitre 4) nous emmène sur le marché de Saint-Pétersbourg. Au milieu du XIX ${ }^{\mathrm{e}}$ siècle, la ville ouvre une nouvelle structure, le passaž, galerie marchande qui remplace les traditionnels bazars. Par l'organisation de cet espace où circulent les corps, les autorités construisent une modernité où disparaissent les mauvaises odeurs et sont offertes de nouvelles sensations visuelles, mais également régissent les règles des rapports entre hommes et femmes. Le régime soviétique n'est pas en reste et s'attelle au goût. La contribution d'Anton Masterovoy (chapitre 8) revient sur les différentes campagnes du gouvernement, durant l'ensemble de la période, pour « changer le menu traditionnel» (p. 167). Selon l'auteur, les gouvernants, tout comme les scientifiques, cherchent à ajuster les préférences gustatives des citoyens à l'économie de déficit et construisent la définition des goûts en opposition à l'Ouest. Mais l'ingéniérie sociale s'exprime également dans la gestion de groupes particuliers, comme le montre Claire Shaw (chapitre 9) qui se penche sur les politiques soviétiques concernant les sourds. Dans une société qui met en avant l'oralité, l'auteure analyse la construction de la communauté des sourds en URSS et les politiques développées par le gouvernement pour l'insertion de cette dernière dans la société - ce qui ne s'est pas fait sans tensions -, et pour transformer les sourds en « hommes nouveaux ».

Enfin, un troisième groupe de contributions entreprend de revoir l'histoire des guerres russes par les sens des acteurs historiques. Si la recherche sur les expériences de guerre a démontré le rôle de la subjectivité et du corps des individus sur le front, ici, les auteurs montrent qu'un pas supplémentaire peut être fait pour saisir cette expérience. Laurie S. Stoff (chapitre 6) se penche sur les sens des infirmières de l'armée russe durant la Première Guerre mondiale : elles touchent des corps blessés, voient des atrocités, entendent des sons inhabituels. Leur expérience n'est pas si différente de celle des soldats, mais est marquée par leur socialisation de genre (p. 120). Steven G. Jug (chapitre 10), lui, se propose d'examiner la construction de l'expérience sensorielle des soldats lors de la Seconde Guerre mondiale, en l'inscrivant dans un contexte culturel soviétique, tout en y analysant ce qui la distingue, notamment de l'image du héros désincarné proposé par les autorités. Adrienne Harris (chapitre 11), elle, propose une réflexion sur les sens et la construction du martyre dans la mémoire collective de la Seconde Guerre mondiale, en revenant sur l'exécution de la jeune 
komsomol Zoja Kosmodemjanskaja. L'auteure démontre que les sens ont participé à faire de cette mort, grâce à une photographie de son cadavre gelé largement diffusée, un récit marquant de la mémoire collective sur la guerre, épisode dont la description détaillée permet une «re-perception » par ses récipiendaires. Toujours sur la construction de la mémoire collective de la guerre, mais aussi du stalinisme, Tim Harte (chapitre 12) analyse le rôle des odeurs dans les films d'Aleksej German, la mise en scène des mauvaises odeurs servant à décrire le passé soviétique. Sans traiter de la guerre, mais abordant un problème prégnant de cette dernière, la contribution de Matthew P. Romaniello (chapitre 2) aborde une question qui occupait déjà les étrangers en Russie : le froid. En s'appuyant sur les discours médicaux des scientifiques occidentaux qui travaillaient à l'Académie des sciences, l'auteur revient sur le développement de la théorie des humeurs, appliquée à un climat froid. L'analysant comme un outil important pour les scientifiques afin de comprendre les effets du froid sur leur propre corps (p. 38), il montre ainsi que l'image si peu positive du froid russe est une construction culturelle et a des racines profondément ancrées dans l'histoire.

À la fin de ce voyage sensitif d'une très grande richesse, il ne fait plus de doute que saisir les sens offre une occasion historiographique de mieux comprendre l'histoire culturelle de la Russie. Les contributions montrent de manière tout fait convaincante et originale que la dimension sensorielle permet de porter un regard renouvelé sur la culture, mais également sur d'autres domaines de la société russe : l'économie, le politique, l'urbanisme, la médecine, entre autres. En réfléchissant par les sens sur les identités et les politiques menées ces trois derniers siècles, on peut désormais parler également d'une construction de « communautés sensitives ». S'il fallait apporter une critique à cet ouvrage très complet, et elle est bien mince par rapport aux apports tout à fait innovants, nous pourrions noter qu'une brillante introduction fait œuvre également de synthèse bienvenue, qui donne une cohérence que le seul découpage chronologique n'autorise pas. Une introduction au début de chaque partie pour présenter les caractéristiques propres à chacune des époques aurait été opportune. Cette lecture, toujours plaisante, est à conseiller à toute personne désirant « sentir » le passé russe et s'adresse également à un plus large public que le seul historien.

1 -Alain Corbin, Le miasme et la jonquille: l'odorat et l'imaginaire social, $X V I I I^{e}-X I X^{e}$ siècles, P. : Flammarion, 1982, coll. " Champs ».

2 - Constance Classen, ed., A Cultural History of the Senses, Bloomsbury Academic, 2014, The Cultural Histories Series.

3 - Ils ont publié en commun sur l'histoire du tabac en Russie : Matthew P. Romaniello and Tricia Starks eds., Tobacco in Russian History and Culture: From the Seventeenth Century to the Present, New York : Routledge, 2009.

4 - Dans le même ordre d'idées et également sur la question des sens, nous pouvons aussi citer le projet de recherche du Professeur Igor' Narskij « Russische Revolution, Migration und Hygiene: Perspektiven und Probleme einer sowjetischen Kulturgeschichte von Gerüchen [Révolution russe, migration et hygiène : perspectives et problèmes d'une histoire culturelle soviétique des odeurs] ».

\section{Magali Delaloye}

Université de Lausanne 